\title{
LA EDUCACIÓN PARA LA SALUD COMO RECURSO PARA AFRONTAR LA COVID-19
}

\author{
Carmen Vizoso-Gómez \\ Universidad de León
}

RESUMEN: La educación para la salud se presenta como un recurso para la promoción de hábitos de conducta que permiten resolver los problemas de salud, desarrollar el bienestar y prevenir la aparición y el contagio de enfermedades. Por su parte, la alfabetización en salud implica tanto la habilidad para entender cuestiones relacionadas con la salud como la motivación para actuar de forma consciente y responsable en beneficio de la salud propia y la colectiva. El objetivo principal de este artículo es analizar diversas iniciativas de educación para la salud en las que se trata de enseñar a afrontar la situación actual provocada por la COVID-19 a personas de diferentes edades. Para ello, se ha empleado una metodología exploratoria, descriptiva y explicativa. Los resultados de los estudios analizados permiten extraer algunas orientaciones para planificar estrategias de promoción de la salud. Se concluye que se deben realizar campañas de alfabetización en salud, utilizando diversos medios de comunicación, para informar y motivar a toda la población sobre la importancia de adoptar comportamientos para la prevención del contagio de la COVID-19 y de mantener hábitos de vida saludables.

PALABRAS CLAVE: educación para la salud, alfabetización en salud, promoción de la salud, covid-19.

\section{HEALTH EDUCATION AS A RESOURCE TO COPE WITH COVID-19}

ABSTRACT: Health education is presented as a resource for the promotion of behavioral habits that allow dealing with health problems, developing well-being and preventing the appearance and spread of diseases. Besides, health literacy implies both the ability to understand health-related issues and the motivation to act consciously and responsibly for the benefit of personal and collective health. The objective of this article is to analyze various health education initiatives in which the aim is to teach people of different ages to face the current situation caused by the COVID-19. An exploratory, descriptive and explicative methodology was followed. In addition, some guidelines are 
proposed for health promotion before, during and after a pandemic. The results of the analyzed studies allow to extract some guidelines for planning health promotion strategies. It is concluded that health literacy campaigns should be carried out, using various media, to inform and motivate the entire population about the importance of adopting behaviors for the prevention of the spread of COVID-19 and maintaining healthy lifestyle habits.

KEYWORDS: Health education, health literacy, health promotion, covid-19.

Recibido: $31 / 07 / 2020$

Aceptado: 12/02/2021

Correspondencia: Carmen Vizoso Gómez, Facultad de Educación, Universidad de León, Campus de Vegazana, 24071 León. Email: cvizg@unileon.es

\section{INTRODUCCIÓN}

\subsection{Promoción, educación y alfabetización en salud}

La educación para la salud se presenta como una estrategia o un recurso para la enseñanza y aprendizaje de conceptos relacionados con la salud (y la enfermedad) y de habilidades que permiten el desarrollo del bienestar tanto individual como comunitario (Salvador y Suelves, 2009). Según la Organización Mundial de la Salud (OMS), mediante la educación para la salud se trata de instruir a las personas para que sean capaces de identificar y afrontar sus problemas de salud y sus necesidades con sus propios recursos y/o, si es necesario, con ayuda externa, desarrollando así una vida sana y, en definitiva, el bienestar de toda la sociedad (OMS, 1989, 2016). Por su parte, Perea (2009) destaca que la educación para la salud ha de ser un proceso de formación permanente, continuo, desarrollado a lo largo de todo el ciclo vital, lo que permite que las personas adquieran hábitos de vida saludables y participen en el progreso de la salud colectiva, yendo más allá del simple objetivo de evitar la aparición de enfermedades.

Así pues, la educación para la salud es un recurso indispensable para el desarrollo o promoción de la salud en la ciudadanía. En este sentido, la OMS postula, en la Carta de Ottawa, que la promoción de la salud es un proceso mediante el cual se aportan los recursos para mejorar la salud de las personas y para que, en última instancia, éstas puedan controlarla, incrementarla y fomentarla (OMS, 1986, 2016). De este modo, los proyectos de promoción de la salud, mediante la educación para la salud y el desarrollo de políticas públicas saludables, deben adaptarse a las características de cada grupo social en el que se vayan a implementar y de las necesidades específicas que presente en cada momento histórico (Bodkin y Hakimi, 2020).

Con el fin de obtener los mejores resultados, la educación para la salud se debería Ilevar a cabo por profesionales que traten de promover la salud a cualquier nivel (comunitario, grupal o individual), entre los que se puede destacar especialmente a los educadores, pedagogos, psicólogos, biólogos, trabajadores sociales y a los sanitarios (Pérez-González et al., 2020). 
Hay que mencionar, además, que a través de la educación para la salud se pretende conseguir la alfabetización en salud (ver figura 1). En concreto, la alfabetización en salud "conlleva el conocimiento, la motivación y las competencias para acceder, comprender y emplear la información, para hacer valoraciones y tomar decisiones sobre temas cotidianos de salud, prevención de enfermedades y fomento de la salud" (Sørensenn et al., 2015, p. 1). Por lo tanto, esta alfabetización implica un proceso más complejo que la mera habilidad para entender cuestiones relacionadas con la salud porque supone también el compromiso de actuar de forma consciente y responsable en beneficio de la salud propia y la colectiva. Para conseguir la alfabetización en salud, Gavidia et al. (2019) consideran que desde la educación para la salud se debería trabajar una competencia general de salud que implica la capacidad y la disposición para emplear los recursos personales (conocimientos, actitudes, habilidades, etc.) que permiten afrontar los problemas de salud y crear un entorno saludable. En definitiva, se podría establecer que la finalidad última de la educación para la salud y de la alfabetización en salud consistiría en mejorar o al menos mantener la calidad de vida y el bienestar de las personas (Juvinyà-Canal et al., 2018).

Figura 1. Relación entre promoción, educación y alfabetización en salud

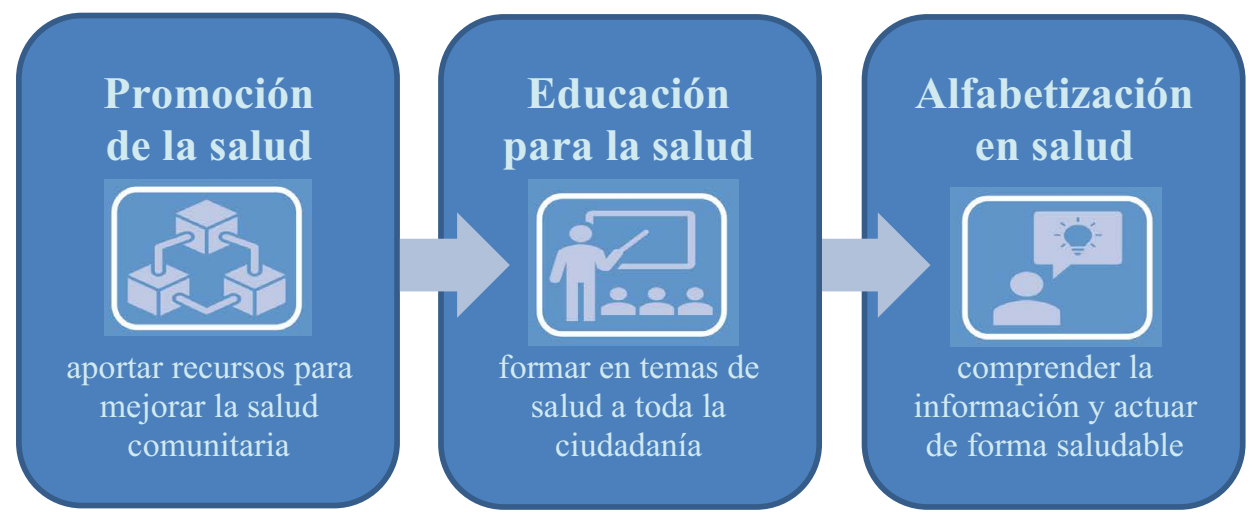

En términos generales, en el desarrollo de programas de educación y alfabetización en salud se deberían emplear estrategias dialógicas y participativas para potenciar el desarrollo de valores tan esenciales como el respeto a la dignidad de la persona, la responsabilidad y la solidaridad con los demás y con las futuras generaciones (Bodkin y Hakimi, 2020; Escámez, 2001). En esta línea, Nutbeam $(2015,2019)$ establece que las estrategias de alfabetización en salud deben guiarse por la utilización de una comunicación que propicie la interacción, la participación y el análisis crítico, especialmente en las iniciativas que abordan la educación para la salud en las escuelas.

\subsection{Educación para la salud en contextos educativos}

En España, la educación para la salud y el desarrollo de hábitos de vida saludables están amparados por la legislación educativa desde la aparición de la Ley Orgánica 1/1990 de Ordenación General del Sistema Educativo (LOGSE) hasta la vigente 
Ley Orgánica 8/2013 para la mejora de la calidad educativa (LOMCE). En líneas generales, desde las diferentes leyes se establece que los escolares que cursan las etapas educativas obligatorias deben alcanzar un desarrollo adecuado que favorezca el logro de una vida activa, saludable y autónoma. En concreto, actualmente se defiende que el alumnado debe desarrollar una serie competencias saludables que le permitirían resolver los problemas de salud en diferentes ámbitos (Ayuso et al., 2019; Gavidia et al., 2019; OMS, 2016). Las principales competencias a desarrollar se describen en la tabla 1.

Tabla 1. Competencias saludables para cada ámbito de salud

\begin{tabular}{|l|l|}
\hline Ámbito & Competencia saludable \\
\hline $\begin{array}{l}\text { Promoción de la } \\
\text { salud }\end{array}$ & $\begin{array}{l}\text { Capacidad y predisposición para controlar los factores que } \\
\text { intervienen en la salud personal y social }\end{array}$ \\
\hline Higiene & $\begin{array}{l}\text { Capacidad para desarrollar y mantener pautas de higiene diaria que } \\
\text { prevengan la aparición de enfermedades y mejoren la calidad de } \\
\text { vida }\end{array}$ \\
\hline Alimentación & Capacidad para Ilevar una alimentación saludable \\
\hline Actividad física & Capacidad para desarrollar una actividad física adecuada \\
\hline Descanso & Capacidad para desarrollar pautas de descanso y sueño saludables \\
\hline Adicción & Capacidad para detectar y prevenir conductas adictivas \\
\hline Afectivo-sexual & $\begin{array}{l}\text { Capacidad para desarrollar una conducta sexual placentera y } \\
\text { saludable }\end{array}$ \\
\hline Mental y emocional & $\begin{array}{l}\text { Capacidad para detectar y combatir situaciones que generen } \\
\text { ansiedad, estrés o depresión y capacidad para gestionar las } \\
\text { emociones }\end{array}$ \\
\hline Social & $\begin{array}{l}\text { Capacidad para establecer y desarrollar relaciones sociales } \\
\text { saludables }\end{array}$ \\
\hline Seguridad & $\begin{array}{l}\text { Capacidad para prevenir los riesgos y para evitar o reducir los } \\
\text { accidentes }\end{array}$ \\
\hline Ambiental & Capacidad para contribuir a la creación de un ambiente saludable \\
\hline
\end{tabular}

Fuente: Ayuso et al., 2019; Gavidia et al., 2019

Por tanto, los centros educativos se presentan como un contexto propicio para la promoción de la salud mediante la educación para la salud que debería comprender todos los contenidos de las disciplinas del currículo desde una perspectiva transversal, dada su relevancia para el bien social (Monsalve, 2014). De este modo, al incluir la educación para la salud en el currículo, se propicia la difusión de información, la formación, la prevención y la promoción de actitudes y habilidades saludables en el alumnado (Manjón y Carrasco, 2007; Ayuso et al., 2019). Al mismo tiempo, para poder fomentar el desarrollo de estas escuelas promotoras de salud, es necesario realizar una adecuada formación del profesorado, considerando el diseño de actividades didácticas, la preparación de recursos educativos y la elaboración de las programaciones pertinentes, contando siempre con la colaboración de otros agentes socializadores como las familias y los profesionales sanitarios (Talavera y Gavidia, 2013). Además, el hecho de incorporar enfermerías escolares en los centros educati- 
vos permite llevar a cabo intervenciones que implican a personal de enfermería para promover el desarrollo de competencias en salud en toda la comunidad educativa (Álvarez et al., 2018).

En los últimos años se han propuesto múltiples programas enfocados a la educación para la salud en las etapas de Educación Infantil y Primaria desarrollados en escuelas españolas, aunque en la mayoría de los casos se orientan a la prevención de enfermedades más que a fomentar la salud (Guerrero-Ramos, 2014). También en la etapa de Educación Secundaria se ha abordado la educación para la salud con el objetivo de promover conductas saludables en los adolescentes (Azorín, 2016; Vega et al., 2015). No obstante, a pesar de estas iniciativas programadas para promocionar la salud, es necesario diseñar nuevas propuestas de competencias en salud que suplan los déficits que presenta actualmente el currículo prescriptivo de la educación obligatoria (Ayuso et al., 2019; Gavidia et al., 2019).

Por último, Torres-García y Santana-Hernández (2017) defienden que también en los grados y másteres de los ámbitos de la Educación Superior se debe contemplar una formación adecuada en promoción y educación para la salud, siguiendo las directrices del Espacio Europeo de Educación Superior (EEES). Asimismo, LlorentBedmar y Cobano-Delgado (2019) proponen que la formación del profesorado debe incluir una educación para la salud apropiada, integral y obligatoria en los curricula de los grados universitarios, donde se aborde la promoción de hábitos de vida saludables que fomentan el equilibrio entre los aspectos físicos, mentales y sociales que engloban la salud. De este modo, los docentes que reciban la formación oportuna podrán educar en salud a sus alumnos y convertir los centros educativos en escuelas promotoras de salud, lo que se traducirá en una mejora en la calidad de vida de los ciudadanos (OMS, 2018).

\subsection{Educación para la salud en adultos}

Cabe destacar la importancia de la educación y alfabetización en materia de salud como parte de la educación permanente de adultos. En este sentido, Lopes y McKay (2020) establecen que la educación y la alfabetización en salud se deben promover, no solo durante los años de escolarización obligatoria o de estudios reglados superiores, sino a lo largo de todo el ciclo vital, involucrando especialmente a las personas que tienen mayores dificultades para acceder al sistema sanitario. Por consiguiente, la educación para la salud debería abarcar la educación formal, no formal e informal. Esto propiciaría que la ciudadanía adquiriese y actualizase los conocimientos y habilidades necesarios para llevar a cabo las conductas más adecuadas en cada situación, lo que les permitiría estar protegidos y proteger al resto de la sociedad frente a posibles riesgos sanitarios.

En los últimos años, las principales iniciativas que se han desarrollado para fomentar la promoción y la alfabetización en salud se han llevado a cabo a través de las Tecnologías de la Información y la Comunicación (TIC), lo que ha recibido el nombre de alfabetización electrónica en salud o alfabetización en e-salud (Norman, 2011). Así, se ha comprobado que este tipo de alfabetización puede ser eficaz en personas de cualquier edad, desde jóvenes hasta ancianos (Xie, 2011). La educación para la salud mediante esta nueva vía permite que la información sobre salud se actualice 
continuamente, esté siempre accesible y pueda difundirse rápidamente. No obstante, es necesario controlar que la información transmitida sea veraz ya que, en caso contrario, se puede fomentar la aparición de teorías ficticias y conspiratorias que incitan a las personas a adoptar patrones de conducta inadecuados y peligrosos al aceptar como válidos los contenidos erróneos (Chong et al., 2020).

Por lo tanto, resulta de suma importancia elaborar políticas y estrategias nacionales e institucionales para la promoción de la alfabetización en salud (Trezona et al., 2018). En este sentido, desde la Unión Europea se han establecido iniciativas para fomentar esta alfabetización, entre los que destaca la labor desempeñada en ciertos países como España, Italia y Portugal, donde se han descrito políticas que se implementan a nivel nacional, regional y local (Heijmans et al., 2015). A modo de ejemplo, en España se podría destacar la Estrategia de promoción de la salud y prevención en el Sistema Nacional de Salud (Ministerio de Sanidad, Servicios Sociales e Igualdad, 2014), que ha dado lugar a la creación de la web Estilos de vida saludable.

En suma, la educación para la salud supone un recurso para fomentar la promoción y alfabetización en cuanto a salud se refiere mediante programas dirigidos a personas de todas las edades, propiciando el bienestar de cada individuo y, en definitiva, de la sociedad.

\section{La PaNDEMIa de la COVID-19}

\subsection{Descripción de la COVID-19}

Uno de los mayores problemas de salud en la historia reciente es la denominada coronavirus disease 2019 (COVID-19), provocada por el virus SARS-CoV-2 y declarada pandemia a nivel mundial por la OMS el día 11 de marzo de 2020 tras comprobar los altos niveles de gravedad y propagación de esta enfermedad (OMS, 2020). Actualmente, a 30 de julio de 2020, según informa el Ministerio de Sanidad, Consumo y Bienestar Social en su página web (www.mscbs.gob.es), el número de personas contagiadas por COVID-19 es de 16.523.815 en el mundo, de las cuales 3.068.576 se han registrado en Europa y, más concretamente 282.641 son los casos registrados en España.

La infección por COVID-19, tras un período de unos 5 días de incubación, puede conllevar una serie de síntomas entre los que destacan los siguientes: fiebre, tos seca, dificultad para respirar y fatiga (Rothan y Byrareddy, 2020). Además, las personas que sufren esta enfermedad pueden presentar otros síntomas menos frecuentes, como son la disnea, producción de esputo, hemoptisis, dolor de cabeza, diarrea y linfopenia. Si los síntomas persisten y se agravan, la enfermedad puede causar la muerte del paciente, lo que suele ocurrir tras una media de 14 días después de la aparición de las primeras manifestaciones (Wang et al., 2020).

Las personas con mayor riesgo de fallecer tras contraer la enfermedad son aquellas que antes del contagio presentaban ciertas patologías, como por ejemplo hipertensión arterial, enfermedades cardiovasculares, enfermedad respiratoria crónica, diabetes mellitus o algún tipo de cáncer (Zhou et al., 2020). Además, se ha constatado que la edad tiene un efecto determinante sobre la mortalidad, registrándose una especial vulnerabilidad a partir de los 60 años (Bonanad et al., 2020). 
No obstante, algunas de las personas infectadas por este coronavirus son asintomáticas, es decir, no presentan síntoma alguno, y esto puede hacer que contagien a otros individuos sin siquiera ser conscientes de estar enfermas, ya que la COVID-19 se transmite fundamentalmente por contacto directo entre personas (Rothan y Byrareddy, 2020).

Por otra parte, la percepción de amenaza de contagio que conlleva la COVID-19 puede afectar a la salud mental y al estado emocional de las personas que no han sido contagiadas. Así, se ha evidenciado que, en España, durante el confinamiento decretado por el gobierno, las personas que sentían una mayor amenaza de ser contagiadas presentaban estados de ánimo negativos y signos emocionales de tristeza, depresión, ansiedad y hostilidad o enojo (Pérez-Fuentes et al., 2020).

Además, las medidas de seguridad recomendadas por las autoridades, como mantener la distancia física, evitar el contacto con otras personas y, sobre todo, el confinamiento, pueden llevar unidas serias consecuencias para el bienestar de la población porque en muchas ocasiones se asocian a la soledad, al aislamiento y a carencias en el apoyo social que derivan en un deterioro de la salud física, mental y social (Saltzman et al., 2020).

Teniendo en cuenta los aspectos descritos, se hace evidente la necesidad de emprender acciones de educación para la salud para prevenir la propagación de la enfermedad y evitar el deterioro de la salud y el bienestar físico, emocional y social de los ciudadanos.

\subsection{Prevención del contagio de la COVID-19}

Con el fin de frenar el avance de la pandemia, el Gobierno de España, a través de la página web del Ministerio de Sanidad, Consumo y Bienestar Social, mantiene un canal de información sobre la COVID-19 dirigido al conjunto de la ciudadanía. Se puede destacar, en este caso, las indicaciones que se presentan para tratar de frenar la transmisión de la enfermedad (Secretaría General de Sanidad, 2020): utilizar una mascarilla que cubra boca y nariz; utilizar pañuelos desechables y lavar las manos inmediatamente después; cubrir la boca y la nariz con el codo flexionado al toser y al estornudar; lavar las manos frecuentemente con agua y jabón o soluciones alcohólicas; evitar tocar los ojos, la boca y la nariz con las manos para evitar la transmisión si las manos no están desinfectadas; mantener siempre limpias y desinfectadas las superficies que más se tocan, y guardar una distancia física de seguridad de al menos un metro y medio con respecto a las demás personas. Además, en cuanto aparezca alguno de los síntomas asociados a la enfermedad (fiebre o tos), lo indicado es aislarse en el domicilio y contactar telefónicamente con los servicios de salud.

Hay que tener en cuenta, no obstante, que para educar, concienciar y motivar a la población para que realice los comportamientos saludables adecuados ante una emergencia tan grave como la actual es imprescindible difundir información veraz y contrastada, pero esto puede no ser suficiente. También es importante identificar y desmentir la información falsa que se pueda transmitir desde ciertos medios. En este sentido, se advierte que, junto a la pandemia generada por el coronavirus, ha aparecido una infodemia, es decir, una propagación acelerada de noticias falsas, información engañosa y bulos a través de medios de comunicación electrónicos, espe- 
cialmente de las plataformas de redes sociales (Chong et al., 2020; García-Marín, 2020). Por todo ello, se hace evidente la relevancia de la educación para la salud y la alfabetización como herramientas fundamentales e imprescindibles con el objetivo de reducir los efectos de la pandemia y de la infodemia en la salud de los ciudadanos y conseguir que éstos accedan a información de calidad y muestren la responsabilidad y la intención de llevar a la práctica inequívocamente las conductas oportunas para reducir la transmisión de la enfermedad.

\section{EducaCión, alfabetización y promoción de la salud frente a la COVID-19}

\subsection{Educación para la salud frente a la COVID-19 en centros educativos}

Como ya se ha reflejado con anterioridad, la educación para la salud ha de permitir la alfabetización y la promoción de la salud en toda la población para mejorar el bienestar y la calidad de vida de cada individuo particular y de la sociedad en general. La educación para la salud, impartida a través de contextos propiamente educativos, como son las escuelas, permite que se transmita información fiable y objetiva a los educandos y, por consiguiente, se evita que sean víctimas de la desinformación. En particular, Guan et al. (2020) justifican la educación y promoción de la salud entre los estudiantes en edad escolar, en defensa de su derecho a una vida sana y activa. Así, se refieren a la relevancia de promover iniciativas relacionadas con el desarrollo de hábitos de vida saludables que implican a múltiples agentes: educadores, profesores, progenitores, cuidadores, profesionales de la salud, medios de comunicación y al propio alumnado. Además, establecen que los gobiernos deberían promover comportamientos saludables a través de mensajes públicos contando con la participación de personajes de prestigio para el alumnado.

En este sentido, Gray et al. (2020) proponen la elaboración de una intervención dirigida a escolares para implementar en los centros educativos. Estos autores describen el valor de los vídeos de dibujos animados, de las películas y de los programas de televisión a la hora de transmitir información en los proyectos de educación para la salud porque suponen una vía que capta la atención de los pequeños. En concreto, la propuesta incluye la realización de varias tareas: la visualización de un video de dibujos animados, el desarrollo de debates en el aula para analizar la información transmitida y la programación de concursos de dibujo y ensayo sobre el tema tratado. Estas tareas tendrían el objetivo de abordar la importancia de la higiene y de las principales conductas de prevención, que en este caso serían el lavado de manos, la precaución al toser y estornudar, el uso de pañuelos desechables y el mantenimiento de la distancia física respecto a otras personas. Con este tipo de intervenciones, los autores consideran que se puede ayudar a minimizar el miedo y la ansiedad ante la COVID-19, especialmente en los niños de menor edad.

En la misma línea, Álvarez (2020) reflexiona sobre los efectos del confinamiento en los estudiantes que no han podido asistir a los centros educativos y plantea que, cuando se retomen las clases presenciales en los centros escolares, se deberían llevar a cabo una serie de intervenciones socioeducativas para tratar de mejorar su bienestar. Así, entre las actuaciones que propone este autor se puede destacar las dirigidas 
a fomentar la educación para la salud, haciendo incidencia en abordar el miedo y la ansiedad ante la pandemia, en tratar las posibles fobias que se hayan podido desarrollar y en superar los efectos del duelo por haber perdido a algún ser querido. En definitiva, se trataría de orientar a los niños y adolescentes para que puedan afrontar esta nueva situación vital de forma adaptativa.

Por último, Andrews et al. (2020) inciden en la importancia que supone concienciar a los jóvenes en el mantenimiento de la distancia personal para prevenir los contagios entre ellos y, posteriormente, entre otras personas de su entorno. Bien es sabido que durante la adolescencia y la juventud se suele experimentar una alta necesidad de interacción social con los pares, que se tiende a asumir riesgos y llevar a cabo conductas imprudentes y, además, se suele buscar el reconocimiento y la aprobación de los amigos. No obstante, los autores apuntan que este último factor, el efecto de la influencia de los pares, podría aprovecharse para que las pandillas asumiesen en grupo las medidas de distancia social, utilizando las redes sociales. Además, esta medida podría ser más eficaz si se involucrase a los personajes influyentes o 'influencers' en la promoción de conductas saludables como el distanciamiento físico.

\subsection{Educación para la salud frente a la COVID-19 más allá del contexto educativo}

Es preciso tener presente que la educación para la salud debe ir dirigida a personas de todas las edades y en diferentes contextos, no solamente se ha de impartir en las escuelas, para lograr alfabetizar en salud a buena parte de la sociedad (Lopes y McKay, 2020). En este sentido, Nutbean (2019) defiende que la promoción de la salud actualmente se ha de construir a partir del acceso universal a una educación sanitaria íntegra y permanente. Además, Harnett (2020) sentencia que la alfabetización en salud debe representar una de las principales estrategias puestas en marcha desde los servicios públicos como respuesta a la planificación de desastres en general y como intervención durante la situación de emergencia sanitaria ligada a la pandemia por coronavirus en particular.

Tradicionalmente, siguiendo las recomendaciones de la OMS (1989, 2016, 2018) en los programas de educación para la salud se han utilizado múltiples métodos y medios para transmitir la información, como por ejemplo el estudio de casos, los debates sobre salud, los carteles, las películas, las noticias en medios de comunicación, etc. No obstante, en los últimos años, tras la aparición de las TIC, buena parte de la información sobre salud se difunde a través de internet. Teniendo en cuenta que durante los peores momentos de la pandemia buena parte de la población fue confinada en sus hogares, el uso de internet en general y de las redes sociales en particular se generalizó para relacionarse con otras personas y también para obtener información sobre temas relacionados con la salud y con el coronavirus (Harnett, 2020). Por ello, ahora se hace indispensable la alfabetización electrónica/digital en salud o alfabetización en e-salud (Norman, 2011). En concreto, esta alfabetización se asocia con la adquisición, el análisis y la aplicación de la información, y conlleva un cambio en los conocimientos, las actitudes y los comportamientos (Truman et al., 2020). Esto implica que los ciudadanos que desarrollen una completa alfabetización en salud serán capaces de aprehender la información y utilizarla para proceder de forma apropiada y oportuna. 
En esta línea, Vandormael et al. (2020) propusieron una intervención para la promoción y educación para la salud de personas adultas basada en el visionado de un video que está disponible en internet y que fue ideado para mejorar los hábitos de higiene saludables durante la crisis generada por la Covid-19. Las conductas planteadas son el lavado de manos, el respeto de la distancia interpersonal, la limpieza de superficies en el hogar, el uso intransferible de utensilios para comer o beber y el control del impulso de acumular productos de primera necesidad. El objetivo de dicha intervención fue ampliar los conocimientos de la población sobre higiene relacionados con el virus y, además, tratar de incrementar la intención de reproducir las conductas higiénicas en situaciones de la vida cotidiana.

Una iniciativa similar, orientada a personas mayores de 65 años, se centra en la promoción de hábitos saludables durante la pandemia (Aung et al., 2020). En este caso, se presenta la difusión de un video, que podría ser descargado en tabletas o en los teléfonos móviles, para potenciar la realización de ejercicio físico en el hogar. Además, se propone la alternativa de repartir DVDs o pósteres en los servicios de atención primaria para aquellos mayores que no pudiesen acceder a la información a través de otras vías.

Por otra parte, Vaz de Almeida y Veiga (2020) exponen que los Centros de Día se presentan como contextos idóneos para la promoción de la educación y la alfabetización en salud destinada a las personas de mayor edad. Así, las autoras defienden que en estos centros sociales se suelen organizar actividades que estimulan la vida activa y el establecimiento de relaciones sociales saludables entre los mayores que las visitan a diario, lo que incrementa su calidad de vida y su bienestar. De esta forma, durante la situación de crisis sanitaria provocada por la COVID-19 sería especialmente beneficioso que se ofertasen programas de educación para la salud dirigidos a estas personas, involucrando a los cuidadores que trabajan en estos centros.

En definitiva, la educación para la salud se hace ahora imprescindible para afrontar la pandemia desencadenada por la COVID-19 y, por lo tanto, debe estar destinada a todos los ciudadanos, independientemente de su edad, y debe desarrollarse en todos los contextos posibles y utilizando todos los canales disponibles, contando con la colaboración de profesionales de diferentes ámbitos que estén interesados en la promoción de la salud.

\section{Consideraciones finales y CONClusiones}

Teniendo en cuenta lo expuesto, se concluye que la educación para la salud es indispensable para la promoción de la salud y la alfabetización en contenidos de salud, la prevención de enfermedades y el mantenimiento del bienestar y la calidad de vida en toda la población. La importancia de este tipo de educación se hace más patente, si cabe, en los momentos históricos en los que se afrontan crisis sanitarias mundiales como la relacionada con la actual pandemia de la COVID-19.

En la mayoría de los países se están desarrollando iniciativas y programas con el fin de prevenir el contagio de la enfermedad y de mantener los hábitos de vida saludables. No obstante, el número de personas contagiadas sigue creciendo día tras día. En este sentido, se debe tener en cuenta que muchas personas, a pesar de percibir una 
considerable alarma social, no muestran una preocupación personal proporcional y esto puede afectar al control de la pandemia y a la llegada de nuevas olas (de la Vega et al., 2020). Por lo tanto, urge conseguir la alfabetización en salud de los ciudadanos, es decir, lograr que accedan a la información, la comprendan y, sobre todo, actúen en consecuencia, con responsabilidad y en beneficio de la salud propia y la colectiva.

Así pues, a partir de la información revisada, se plantean una serie de consideraciones que se deberían tener en cuenta a la hora de implementar programas de educación para la salud como recurso para afrontar la COVID-19. En primer lugar, los gobiernos y las administraciones deberían promover políticas y campañas para fomentar la educación y alfabetización en salud y en e-salud. Se debe hacer uso de todos los medios de comunicación para transmitir información veraz sobre las medidas de prevención, utilizando mensajes directos, concisos y comprensibles para toda la ciudadanía. Además, las campañas de información y educación serían más eficaces si se implicase a personas prestigiosas e influyentes.

Respecto a las instituciones y organizaciones, como los centros educativos, centros sanitarios, centros de día, etc., deben implementar programas de educación para la salud dirigidos a personas de todas las edades, contando con el trabajo coordinado de profesionales de diferentes ámbitos. Dichos programas deben incluir contenidos que aborden la prevención del contagio de la COVID-19, los riesgos de mantener comportamientos inadecuados y la recuperación física y emocional de las personas que han padecido la enfermedad. Además, se debe velar por el desarrollo de competencias y hábitos de vida saludables en toda la ciudadanía.

En definitiva, en el momento actual se debe impulsar la educación para salud, que debe ser considerada como un recurso indispensable durante y después de la pandemia para tratar de promover y recuperar el bienestar físico, mental y social, además de intentar evitar la propagación de la COVID-19.

\section{ReFERENCIAS BIBLIOGRÁFICAS}

Álvarez, M. (2020). El confinamiento de niñas y niños en España en 2020 por la crisis del COVID-19: Propuestas desde la Educación Social Escolar para la vuelta al centro escolar. Revista de Educación Social, 30, 457-461.

Álvarez, R., Eguilaz, M. y Miguel, S. (2018). La enfermería escolar: un recurso necesario para la comunidad educativa. Contextos Educativos: Revista de Educación, 22, 165-180. https://doi.org/10.18172/con.3374

Andrews, J. L., Foulkes, L. y Blakemore, S. (2020). Peer influence in adolescence: Public-health implications for COVID-19. Trends in Cognitive Sciences, 24(8), 585-587. https://doi.org/10.1016/j.tics.2020.05.001

Aung, M. N., Yuasa, M., Koyanagi, Y., Aung, T. N. N., Moolphate, S., Matsumoto, H. y Yoshioka, T. (2020). Sustainable health promotion for the seniors during COVID-19 outbreak: A lesson from Tokyo. Journal of Infection in Developing Countries, 14(4), 328-331. https://doi.org/10.3855/JIDC.12684

Ayuso, R., Molina, M. C. y Medina, J. L. (2019). La promoción de salud como materia del currículum educativo en el contexto catalán. Tendencias Pedagógicas, 33, 8398. https://doi.org/10.15366/tp2019.33.007 
Azorín, C. M. (2016). ¡Abre los ojos! Un proyecto de mejora educativa para la prevención de drogas en adolescentes. Revista Complutense de Educación, 27(1), 141-159. http://doi.org/10.5209/rev_RCED.2016.v27.n1.45532

Bodkin, A. y Hakimi, S. (2020). Sustainable by design: A systematic review of factors for health promotion program sustainability. BMC Public Health, 20, e964. https:// doi.org/10.1186/s12889-020-09091-9

Bonanad, C., García-Blas, S., Tarazona-Santabalbina, F., Sanchis, J., Bertomeu-González, V., Fácila, L., Ariza, A., Núñez, J. y Cordero, A. (2020). The effect of age on mortality in patients with COVID-19: A meta-analysis with 611,583 subjects. Journal of the American Medical Directors Association, 21(7), 915-918. https://doi.org/10.1016/j.jamda.2020.05.045

Chong, Y.Y., Cheng, H. Y., Chan, H. Y. L., Chien, W. T. y Wong, S. Y. S. (2020). COVID-19 pandemic, infodemic and the role of eHealth literacy. International Journal of Nursing Studies, 108, e103644. https://doi.org/10.1016/j.ijnurstu.2020.103644

de la Vega, R., Ruíz-Barquín, R., Boros, S. y Szabo, A. (2020). Could attitudes toward COVID-19 in Spain render men more vulnerable than women? Global Public Health, 15(9), 1278-1291. https://doi.org/10.1080/17441692.2020.1791212

Escámez, J. (2001). Valores, actitudes y habilidades en la Educación para la salud. Educación XX1, 4, 41-60. https://doi.org/10.5944/educxx1.4.0.363

García-Marín, D. (2020). Infodemia global. Desórdenes informativos, narrativas fake y fact-checking en la crisis de la Covid-19. El profesional de la información, 29(4). https://doi.org/10.3145/epi.2020.jul.11

Gavidia, V., Garzón, A., Talavera, M., Sendra, C. y Mayoral, O. (2019). Alfabetización en salud a través de las competencias. Enseñanza de las Ciencias: Revista de Investigación y Experiencias Didácticas, 37(2), 107-126. https://doi.org/10.5565/rev/ ensciencias. 2628

Gray, D. J., Kurscheid, J., Mationg, M. L., Williams, G. M., Gordon, C., Kelly, M., Wangdi, K. y McManus, D. P. (2020). Health-education to prevent COVID-19 in schoolchildren: A call to action. Infectious Diseases of Poverty, 9, e81. https://doi. org/10.1186/s40249-020-00695-2

Guan, H., Okely, A. D., Aguilar-Farias, N., del Pozo Cruz, B., Draper, C. E., El Hamdouchi, A., Florindo, A. A., Jáuregui, A., Katzmarzyk, P. T., Kontsevaya, A., Löf, M., Park, W., Reilly, J. J., Sharma, D., Tremblay, M. S. y Veldman, S. L. C. (2020). Promoting healthy movement behaviours among children during the COVID-19 pandemic. The Lancet Child and Adolescent Health, 4(6), 416-418. https://doi. org/10.1016/S2352-4642(20)30131-0

Guerrero-Ramos, D., Jiménez-Torres, M. G. y López-Sánchez, M. (2014). Escuela saludable versus protectora de la salud (salugénica). Educar, 50(2), 323-338. https:// doi.org/10.5565/rev/educar.65

Harnett, S. (2020). Health literacy, social media and pandemic planning. Journal of Consumer Health on the Internet, 24(2), 157-162. https://doi.org/10.1080/15398 285.2020.1756677

Heijmans, M., Uiters, E., Rose, T., Hofstede, J., Deville, W., Van der Heide, I., Boshuisen, H. y Rademaker, J. (2015). Study on sound evidence for a better understanding of health literacy in the European Union. Netherlands Institute for Health Services Research. 
Juvinyà-Canal, D., Bertran-Noguer, C. y Suñer-Soler, R. (2018). Alfabetización para la salud, más que información. Gaceta Sanitaria, 32(1), 8-10. https://doi. org/10.1016/j.gaceta.2017.07.005

Ley Orgánica 1 de 1990, de Ordenación General del Sistema Educativo. de 3 de octubre de 1990. Boletín Oficial del Estado núm. 24172. https://www.boe.es/boe/ dias/1990/10/04/pdfs/A28927-28942.pdf

Ley Orgánica 8 de 2013, para la mejora de la calidad educativa, de 9 de diciembre de 2013. Boletín Oficial del Estado, núm. 12886. https://www.boe.es/buscar/ pdf/2013/BOE-A-2013-12886-consolidado.pdf

Lopes, H. y McKay, V. (2020). Adult learning and education as a tool to contain pandemics: The COVID-19 experience. International Review of Education, 66, 575602. https://doi.org/10.1007/s11159-020-09843-0

Llorent-Bedmar, V. y Cobano-Delgado, V. (2019). La formación en educación para la salud del alumnado universitario del grado de Educación Infantil en España. Ciência \& Saúde Coletiva, 24(8), 3067-3078. https://doi.org/10.1590/141381232018248.28642017

Manjón, J. y Carrasco, J. I. (2007). Educación para la salud: hacia un nuevo modelo estructural y de intervención multidisciplinar desde los centros educativos. Revista Fuentes, 7, 107-116.

Ministerio de Sanidad, Servicios Sociales e Igualdad. (2015). Estrategia de promoción de la salud y prevención en el Sistema Nacional de Salud. https:// www.mscbs.gob.es/profesionales/saludPublica/prevPromocion/Estrategia/docs/ EstrategiaPromocionSaludyPrevencionSNS.pdf

Monsalve, L. (2014). Estudio comparado sobre las políticas educativas en educación para la salud en Francia y España. Bordón, 66(3), 91-103.

Norman, C. (2011). eHealth Literacy 2.0: Problems and opportunities with an evolving concept. Journal of Medical Internet Research, 13(4). https://doi.org/10.2196/ jmir.2035

Nutbeam, D. (2015). Defining, measuring and improving health literacy. Health Evaluation and Promotion, 42(4), 450-456. https://doi.org/10.7143/jhep.42.450

Nutbeam, D. (2019). Health education and health promotion revisited. Health Education Journal, 78(6), 705-709. https://doi.org/10.1177/0017896918770215

OMS (1989). Educación para la salud. Manual sobre educación sanitaria en atención primaria de salud. https://apps.who.int/iris/handle/10665/38660

OMS (1986). Carta de Ottawa para la Promoción de la salud. Conferencia Internacional sobre la Promoción de la Salud. https://iris.paho.org/handle/10665.2/44469

OMS (2016). Shanghai Declaration on promoting health in the 2030 Agenda for Sustainable Development. https:/www.who.int/publications/i/item/WHO-NMHPND-17.5

OMS (2018). Promoting health: guide to national implementation of the Shanghai Declaration. https://www.who.int/publications/i/item/WHO-NMH-PND-18.2

OMS (2020). Alocución de apertura del Director General de la OMS en la rueda de prensa sobre la Covid-19 celebrada el 11 de marzo de 2020. https://www.who. int/es/dg/speeches/detail/who-director-general-s-opening-remarks-at-the-mediabriefing-on-covid-19---11-march-2020 
Perea, R. (2009). Promoción y educación para la salud. Algunos pilares básicos. En R. Perea (Dir.), Promoción y Educación para la Salud. Tendencias innovadoras (pp. 3-26). Ediciones Díaz de Santos.

Pérez-Fuentes M. C., Molero, M. M., Martos, A., Gázquez, J. J. (2020). Threat of COVID-19 and emotional state during quarantine: Positive and negative affect as mediators in a cross-sectional study of the Spanish population. PLOS ONE, 15(6). https://doi.org/10.1371/journal.pone.0235305

Pérez-González, J. C., Yáñez, S., Ortega-Navas, M. C. y Piqueras, J. A. (2020). Educación Emocional en la Educación para la Salud: Cuestión de Salud Pública. Clínica y Salud, 31(3), 127-136. https://doi.org/10.5093/clysa2020a7

Rothan, H. A. y Byrareddy, S. N. (2020). The epidemiology and pathogenesis of coronavirus disease (COVID-19) outbreak. Journal of Autoimmunity, 109, e102433. https://doi.org/10.1016/j.jaut.2020.102433

Saltzman, L. I., Hansel, T. C. y Bordnick, P. S. (2020). Loneliness, isolation, and social support factors in post-COVID-19 mental health. Psychological Trauma: Theory, Research, Practice, and Policy, 12(S1), S55-S57. http://doi.org/10.1037/tra0000703

Salvador, T. y Suelves, J. M. (2009). Ganar salud en la escuela. Guía para conseguirlo. Ministerio de Educación. Ministerio de Sanidad y Política Social. https:// www.mscbs.gob.es/profesionales/saludPublica/prevPromocion/promocion/ saludjovenes/docs/ganarSaludEscuela.pdf

Secretaría General deSanidad (2020). Preguntas y respuestas sobre el nuevo coronavirus (COVID-19). Ministerio de Sanidad. https://www.mscbs.gob.es/profesionales/ saludPublica/ccayes/alertasActual/nCov-China/documentos/20200317_ Preguntas_respuestas_2019-nCoV.pdf

Sørensen, K., Pelikan, J. M., Röthlin, F., Ganahl, K., Slonska, Z., Doyle, G., Fullam, J., Kondilis, B., Agrafiotis, D., Uiters, E., Falcon, M., Mensing, M., Tchamov, K., van den Broucke, S., Brand, H. y on behalf of the HLS-EU Consortium. (2015). Health literacy in Europe: Comparative results of the european health literacy survey (HLS-EU). European Journal of Public Health, 25(6), 1053-1058. https://doi. org/10.1093/eurpub/ckv043

Talavera, M. y Gavidia, V. (2013). Percepción de la educación para la salud en el personal docente y el sanitario. Didáctica de las Ciencias Experimentales y Sociales, 27, 115-129. https://doi.org/10.7203/DCES.27.2569

Torres-García, M. y Santana-Hernández, H. (2017). La Educación para la Salud en la formación de maestros desde el Espacio Europeo de Educación Superior. Revista Complutense de Educación, 28(4), 1083-1101. http://doi.org/10.5209/ RCED.51536

Trezona, A., Rowlands, G. y Nutbeam, D. (2018). Progress in Implementing National Policies and Strategies for Health Literacy. What Have We Learned so Far? International Journal of Environmental Research and Public Health, 15(7), e1554. http:// dx.doi.org/10.3390/ijerph15071554

Truman, E., Bischoff, M. y Elliott, C. (2020). Which literacy for health promotion: Health, food, nutrition or media? Health Promotion International, 35(2), 432-444. https://doi.org/10.1093/heapro/daz007 
Vandormael, A., Adam, M., Greuel, M. y Bärnighausen, T. (2020). A short, animated video to improve good COVID-19 hygiene practices: a structured summary of a study protocol for a randomized controlled trial. Trials, 21(469). https://doi. org/10.1186/s13063-020-04449-1

Vaz de Almeida, C. y Veiga, A. (2020). Social determinants and literacy in health of elderly: Walk to well-being. Open Access Library Journal, 7, e6390. https://doi. org/10.4236/oalib.1106390

Vega, A., Aramendi, P., Buján, M. K. y Garín, S. (2015). La educación para la salud en la ESO: Aportaciones de un estudio sobre el País Vasco. Educación XX1, 18(1), 167-188. http://doi.org/10.5944/educXX1.18.1.12316

Wang, W., Tang, J. y Wei, F. (2020). Updated understanding of the outbreak of 2019 novel coronavirus (2019-nCoV) in Wuhan, China. Journal of Medical Virology, 92(4), 441-447. https://doi.org/10.1002/jmv.25689

Xie, B. (2011). Effects of an eHealth literacy intervention for older adults. Journal of medical Internet research, 13(4), 90. https://doi.org/10.2196/jmir.1880

Zhou, F., Yu, T., Du, R., Fan, G., Liu, Y., Liu, Z., et al. (2020). Clinical course and risk factors for mortality of adult in patients with Covid-19 in Wuhan, China: a retrospective cohort study. Lancet, 395(10229), 1054-1062. https://doi. org/10.1016/S0140-6736(20)30566-3 